\title{
PRODUÇÃO DE PACU EM TANQUES-REDE NO RESERVATÓRIO DEITAIPU, BRASIL: RETORNO ECONÔMICO
}

\author{
ECONOMIC RETURN FROM CAGE PRODUCTION OF \\ PACU IN ITAIPU RESERVOIR, BRAZIL
}

\author{
Silva, J.R. ${ }^{1}$, Rabenschlag, D.R. ${ }^{2}$, Feiden, A. ${ }^{3 A}$, Boscolo, W.R. ${ }^{3 B}$, Signor, A.A. ${ }^{4}$ e Bueno, G.W. ${ }^{5}$ \\ 1UNIOESTE-Universidade Estadual do Oeste do Paraná. Centro de Ciências Sociais Aplicadas. Toledo, PR. \\ Brasil. josemar@unioeste.br \\ 2UFSM-Universidade Federal de Santa Maria. Centro de Tecnologia/CT. Santa Maria, RS. Brasil. \\ denis@ct.ufsm.br \\ ${ }^{3}$ UNIOESTE-Universidade Estadual do Oeste do Paraná. Programa de Pós-Graduação em Recursos

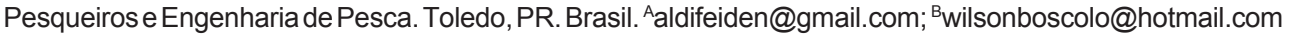 \\ ${ }^{4}$ IFPR-Instituto Federal do Paraná. Setor Aquicultura. Foz do Iguacu-Paraná. Brasil. arcangelo.signor@ \\ ifpr.edu.br \\ ${ }^{5}$ MPA-Ministério da Pesca e Aquicultura. Brasil. guilherme.bueno@mpa.gov.br
}

\section{PalaVRas chaVe adicionais}

Agronegócio. Análise de rentabilidade. Aquicultura. Espécies nativas.

\section{RESUMO}

O objetivo deste trabalho foi avaliar a rentabilidade econômica de um projeto aquícola em tanques-rede para o cultivo de pacu (Piaractus mesopotâmicus) com peso médio final de $1 \mathrm{~kg}$, propiciando assim informações para a tomada de decisão de investidores, produtores, técnicos, órgãos de fomento e demais instituições afetas à área. Para avaliação dos cenários de rentabilidade econômica utilizou-se o método determinístico com auxílio de uma planilha eletrônica. Verificouse que os índices de retorno econômico na produção de pacu em 160 tanques-rede no reservatório de Itaipu apresentaram os piores resultados no cenário I com índice de lucratividade de $-17,22 \%$, devido à conversão alimentar de 3,58 e o preço da ração de $R \$ 0,75 \mathrm{~kg}$. A análise do retorno sobre o investimento (ROI) apresentou os índices de 1,$30 ; 1,36 ; 1,47$ e $1,54 \mathrm{R} \$ / \mathrm{kg}$ entre os cenários $A$ a $D$, respectivamente. $O$ ponto de equilíbrio financeiro médio entre as projeções foi de $\mathrm{R} \$ 226921,1$ e a média do volume mínimo a ser produzido foi de $55286,7 \mathrm{~kg}$ de pacu, baseandose no ponto de equilíbrio físico do projeto com 160 tanques-rede. Para o melhor retorno econômico deve-se produzir no mínimo 40 946,4 kg de pacu

Recibido: 27-4-11. Aceptado: 7-11-11.

\section{AdDitiOnAL KEYWORDS}

Agro business. Profitability analysis. Aquiculture. Native species.

em 160 tanques-rede com uma conversão alimentar de 1:2,49 e preço de venda do peixe a 4,00 atingindo $31,78 \%$ de lucratividade sobre a receita bruta com um ROI de 1,5393 R $\$$ por quilo produzido.

\section{SUMMARY}

The aim of this study was to evaluate the economic profitability a project of aquiculture in cage for the cultivation of pacu (Piaractus mesopotamicus) with an initial medium weight of $1 \mathrm{~kg}$. The study was done to provide information for decision making investors, farmers, technicians, foment organs and other institutions related to the area. To evaluate the economic profitability scenery, a deterministic method, with the help of an electronic spread sheet, was used. It could be verified that the economic outcome indexes in pacu breeding in 160 cages in the Itaipu reservoir, presented the worst results in scenery I with a profitability index of $-17.22 \%$, due to diet conversion of 3.58 and the ration price $(R \$ 0.75 \mathrm{~kg})$. The analysis of return on investment (ROI) presented indexes of $1.30,1.36,1.47$ and $1.54 \mathrm{R} \$ / \mathrm{kg}$ between $A$ and $D$ sceneries, respectively. The medium 


\section{SILVA, RABENSCHLAG, FEIDEN, BOSCOLO, SIGNOR E BUENO}

financial equilibrium point between the projections was of $R \$ 226921.12$ and the average of minimum value to be produced was of $55286.7 \mathrm{~kg}$ pacu, based on the physical equilibrium point of the project with one hundred and sixty net ponds. For a better economic return it is necessary to breed at least $40946.4 \mathrm{~kg}$ pacu in one hundred and sixty net ponds with a diet conversion of 1:2.49 and the fish selling price of 4.00 , reaching a profitability of $31.78 \%$ on the gross revenue with a ROI of 1.54 $\mathrm{R} \$ / \mathrm{kg}$ produced.

\section{INTRODUÇÃO}

O desenvolvimento da atividade pesqueira, para fazer frente à constante elevação da demanda mundial por alimentos, engendrou uma série de políticas que incentivaram a ampliação da produção pesqueira mundial. Estas políticas de incentivo geraram modelos de desenvolvimento da atividade da pesca extrativa, que não pesaram, tampouco se preocuparam com os estoques pesqueiros, levando estes para próximo do limite ou capacidade máxima de exploração sustentável (Ostrensky et al., 2008).

Desta forma, a principal alternativa para suprir o déficit dos estoques pesqueiros está no cultivo de organismos aquáticos, isto pode ser observado com o aumento da produção mundial que obteve um crescimento de 8,4\% em nível mundial (FAO, 2010), destacando-se a piscicultura continental, em diferentes modelos de sistemas produtivos. Segundo o relatório Blue Frontiers: Managing the environmental costs of Aquaculture, a aquicultura é a melhor forma de se produzir proteína animal, do ponto de vista ambiental e econômico. Corroborando com esta afirmativa, a Organização das Nações Unidas para Agricultura e Alimentação considera que as projeções para 2030 no consumo mundial de pescado será em torno de 100 milhões de toneladas por ano.

Neste contexto, o Brasil poderia atender esta demanda e se tornar um dos maiores produtores de peixe do mundo, pois o país possui cerca de 40 espécies de peixes de água doce potenciais de cultivo (Godinho, 2007) e detém cerca de 5,5 milhões de hectares em represas ou reservatórios naturais e artificiais que podem ser utilizados para a prática da aquicultura. Dentre estes reservatórios, na bacia hidrográfica do Paraná III, fronteira entre o Paraguai e Brasil tem-se o reservatório de Itaipu que representa uma área inundada de $1350 \mathrm{~km}^{2}$. Na sua margem brasileira possui cerca de 20 braços, dentre estes, o São Francisco Falso, São Francisco Verdadeiro e Ocoí que foram destinados ao cultivo de pacu (Piaractus mesopotâmicus) em tanques-rede. Nestas áreas aquícolas estimam-se uma produção de 4666 toneladas por ano e a possibilidade de gerar 13211 empregos diretos e 52845 empregos indiretos (MPA, 2010).

O pacu é uma espécie nativa da Bacia da Prata, apresenta maior distribuição nas planícies alagadas da região Centro-Oeste, no Pantanal do Mato Gtosso (Petrere, 1989), destaca-se entre as espécies nativas apresentando desejáveis características zootécnicas para o cultivo como: hábito alimentar onívoro, carne de excelente qualidade e boa aceitação pelos consumidores (Jomori et al., 2003). Rendimento do processamento de $46,73 \%$ de filé sem pele; $16,57 \%$ de cabeça e $88,98 \%$ de rendimento de carcaça segundo Faria et al. (2003), destacando-se como uma interessante alternativa para cultivo. Além de demonstrar uma fácil adaptação à alimentação artificial, elevada rusticidade e fecundidade (Castagnolli e Zuim, 1985).

Portanto, os novos modelos de produção e o crescimento do consumo de peixes promovem o avanço da aquicultura, que por sua vez, demandam informações para tomada de decisão sobre a viabilidade econômica e a rentabilidade dos empreendimentos aquícolas, principalmente no que se refere às espécies de peixes nativos.

Estudos desta natureza vêm sendo desenvolvidos em cultivos de tilápia (Oreochromis niloticus) em reservatórios por Conte (2002); Medeiros (2002); Ono e 
Kubitza (2003); Marengoni e Bueno (2007); Kubitza e Campos (2005); Scorvo-Filho et al. (2006); Furlaneto et al. (2006) e Ayroza (2009). Porém, há uma grande lacuna de informações de projetos de rentabilidade e viabilidade econômica para espécies nativas que também apresentam potencial produtivo, como o pacu (Piaractus mesopotâmicus), jundiá (Rhamdia sp.), surubim (Pseudoplatystoma sp.), entre outras não exóticas.

Entretanto, este trabalho teve como objetivo avaliar os cenários de retorno econômico durante a produção de pacu $(P$. mesopotâmicus) em 160 tanques-rede instalados em uma área aquícola no reservatório de Itaipu.

\section{MATERIALEMÉTODOS}

O trabalho foi realizado durante seis meses em um cultivo experimental de pacu (P. mesopotâmicus) em uma área de pesquisa com tanques-rede, instalada na zona de transição do reservatório da Usina Hidrelétrica Itaipu Binacional, no município de Santa Helena região Oeste do Estado do Paraná, Brasil, com as coordenadas geográficas W54²1'196", S2451'105", W54⒉ $21^{\prime} 078^{\prime \prime}$, S2451'192", e W54'21'224", S24 $51^{\circ} 143^{\prime \prime}$, pertencente à bacia hidrográfica do Paraná III.

Para a avaliação dos índices zootécnicos do pacu, seguiu-se os dados avaliados por Signor et al. (2010) que acompanhou os parâmetros zootécnicos do cultivo. A partir das características técnicas do projeto foram levantadas informações relevantes, as quais fundamentaram o estudo e as projeções da produção e estimativas de custos e receitas.

Para a realização do experimento de avaliação de desempenho dos peixes foram elaboradas seis rações experimentais extrusadas com níveis de $25 \%, 30 \%$ e $35 \%$ de proteína e dois níveis de energia 3000 e 3350 $\mathrm{kcal} / \mathrm{kg}$ de energia digestível, as quais foram cotadas a um custo mínimo de $0,6637 \mathrm{R} \$ / \mathrm{kg}$, custo máximo de $0,7502 \mathrm{R} \$ / \mathrm{kg}$, sendo utili- zado para os cálculos o custo intermediário de $0,7070 \mathrm{R} \$ / \mathrm{kg}$, pois não houve diferenças significativas no desempenho dos peixes alimentados com as diferentes rações.

Os índices zootécnicos foram obtidos de um experimento conduzido em delineamento inteiramente casualizado com seis tratamentos e três repetições totalizando 18 tanques-rede de $5 \mathrm{~m}^{3}$ com densidade de 44 pacus $/ \mathrm{m}^{3}$ com peso médio inicial de 293,38 55,67 g. Em seguida, os cálculos de produção foram estipulados para um empreendimento aquícola com 160 tanquesrede $(1,75 \times 1,75 \times 1,75 \mathrm{~m})$, conforme estabelecido pelo plano diretor da Itaipu Binacional (2007) para produção em uma área aquícola de $6400 \mathrm{~m}^{2}$ no reservatório de forma a atender a capacidade de suporte do local estimada pelo modelo Dillon e Rigler (1974).

O mercado consumidor de destino para a produção foi estabelecido inicialmente como o mercado de varejo, ou seja, feiras livres, peixarias, restaurantes, supermercados e pesque-pague. Neste contexto, considerou-se o preço pago por $\mathrm{kg}$ de pacu vivo proposto por Richter (2004) do Departamento de Economia Rural da Secretaria de Estado Agricultura e Abastecimento (SEAB-DERAL-DEB) no Paraná, o qual considera uma projeção na produção por município do estado e preço médio estadual de comercialização ${ }^{1}$, sendo de R $\$ 2,99 / \mathrm{kg}$ em 2004 , de R $\$ 3,37 / \mathrm{kg}$ em 2005 , de $\mathrm{R} \$ 3,85 / \mathrm{kg}$ em 2006 e de $\mathrm{R} \$ 4,00 / \mathrm{kg}$ em 2007.

De acordo com as condições locais deste estudo, não foi contabilizado a construção de um galpão para armazenamento dos equipamentos e acondicionamento da ração, por já exisitir no local do experimento, não obstante, em outros empreendimento deste formato que não possua local para armazenamento e acondicionamento dos insumos, este item deverá ser considerado nos cálculos do investimento inicial.

Os equipamentos necessários para

${ }^{1} 1,0 \mathrm{US} \$=1,80 \mathrm{R} \$$ 
desenvolvimento da produção, assim como equipamentos para manejo, biometrias e acompanhamento da qualidade da água foram definidos através de pesquisa de campo e consulta as empresas especializadas do ramo.

A depreciação foi calculada pelo método linear, tomando-se o valor do equipamento diminuído do valor residual e dividido pela vida útil.

Os gastos com a adequação das normas e legislação pertinente ao licenciamento, instalação e operação da atividade foram levantadas de acordo com Filipetto (2004) e pesquisas junto ao Instituto Ambiental do Paraná (IAP, 2010) seguindo a Portaria IAP $n^{\circ} 112$, de 13 de julho de 2005.

De posse destes dados, fez-se a simulação dos resultados para diversos cenários de rentabilidade econômica, através de modelos determinísticos em planilhas do Excel ${ }^{\circledR}$. Assim, foram estabelecidos os seguintes cenários possíveis de acordo com a diferenciação nos indicadores/ parâmetros de conversão alimentar (CA), custo de ração $(\mathrm{R} \$ / \mathrm{kg})$, preço de venda do peixe $(\mathrm{R} \$)$ e taxa de sobrevivência $(\%)$. Neste contexto, definiu-se como A e B os cenários mais pessimistas, $\mathrm{C}$ e $\mathrm{D}$ os cenários mais otimistas e, por final, os pessimistas E a J. Esta relação pode ser verificada na tabela II.

Os dados do custo de produção, receitas e montagem do fluxo de caixa foram baseados na estrutura de Custo Operacional (COP) proposta por Matsunaga et al. (1976), orientando assim a aferição de dados primários e, por conseguinte no estabelecimento dos custos de produção. Contudo, foram propostos os seguintes indicadores de rentabilidade do empreendimento: receita bruta $(\mathrm{RB})$ : receita obtida pela venda da produção; lucro bruto $(\mathrm{L})$ : lucro obtido pela diferença entre a RB e o custo total de produção (CT); lucro operacional (LO) ou receita líquida (RL): lucro obtido pela diferença entre receita bruta (RB) e o custo operacional total (COT); margem de contribuição (MC): diferença entre a RB e os custos variáveis (CV); margem bruta do custo total $(\mathrm{MBCT})$ : definida pela relação entre o lucro e custo total de produção; margem bruta do custo operacional total (MBCOT) : relação entre o lucro operacional (ou receita operacional) e custo operacional total de produção; custo médio $(\mathrm{CMe})$ : expresso pela relação entre os custos total de produção, dividido pela quantidade produzida; indice de lucratividade (IL): relação entre o lucro (RB - CT) e demonstra o percentual da receita obtida com a venda da produção; retorno sobre o investimento operacional (RIO): relação entre o lucro operacional pelo investimento e demonstra o percentual da receita obtida com a venda da produção e o ponto de nivelamento ou ponto de equilíbrio físico e financeiro: são indicadores importantes para avaliação econômica da atividade produtiva. Propiciam a determinação da quantidade mínima (Qmín) ou do preço mínimo (Pmín) para cobertura dos custos.

$\mathrm{Na}$ tabela I, verificam-se as projeções dos custos operacionais e o investimento inicial para o projeto de criação de pacu em 160 tanques-rede, onde os itens para investimento inicial foram pesquisados junto às empresas da região oeste do Paraná a fim de efetuar a implantação do projeto em escala econômica para 160 tanques-rede em um ciclo de produção de seis meses para obtenção de um peixe com peso médio de 1 kg.

\section{RESULTADOSEDISCUSSÃO}

Através da simulação do retorno econômico e rentabilidade do cultivo de pacu com densidade de 44 peixes $/ \mathrm{m}^{3}$ em 160 tanques-rede durante 6 meses, obteve-se a produtividade de $75,03 \mathrm{~kg} / \mathrm{m}^{3} /$ ano e no melhor cenário econômico (D) uma lucratividade sobre receita bruta de $31,78 \%$, com índice de lucratividade de $1,31 \mathrm{R} \$ / \mathrm{kg}$ (tabela II). Demonstrando a potencialidade desta espécie para produção em sistema intensivo. Também avaliando o retorno econômico em tanques-rede de $6 \mathrm{~m}^{3}$ no rio Parana- 
RETORNO ECONÔMICO DA PRODUÇÃO DE PACU EM TANQUES-REDE

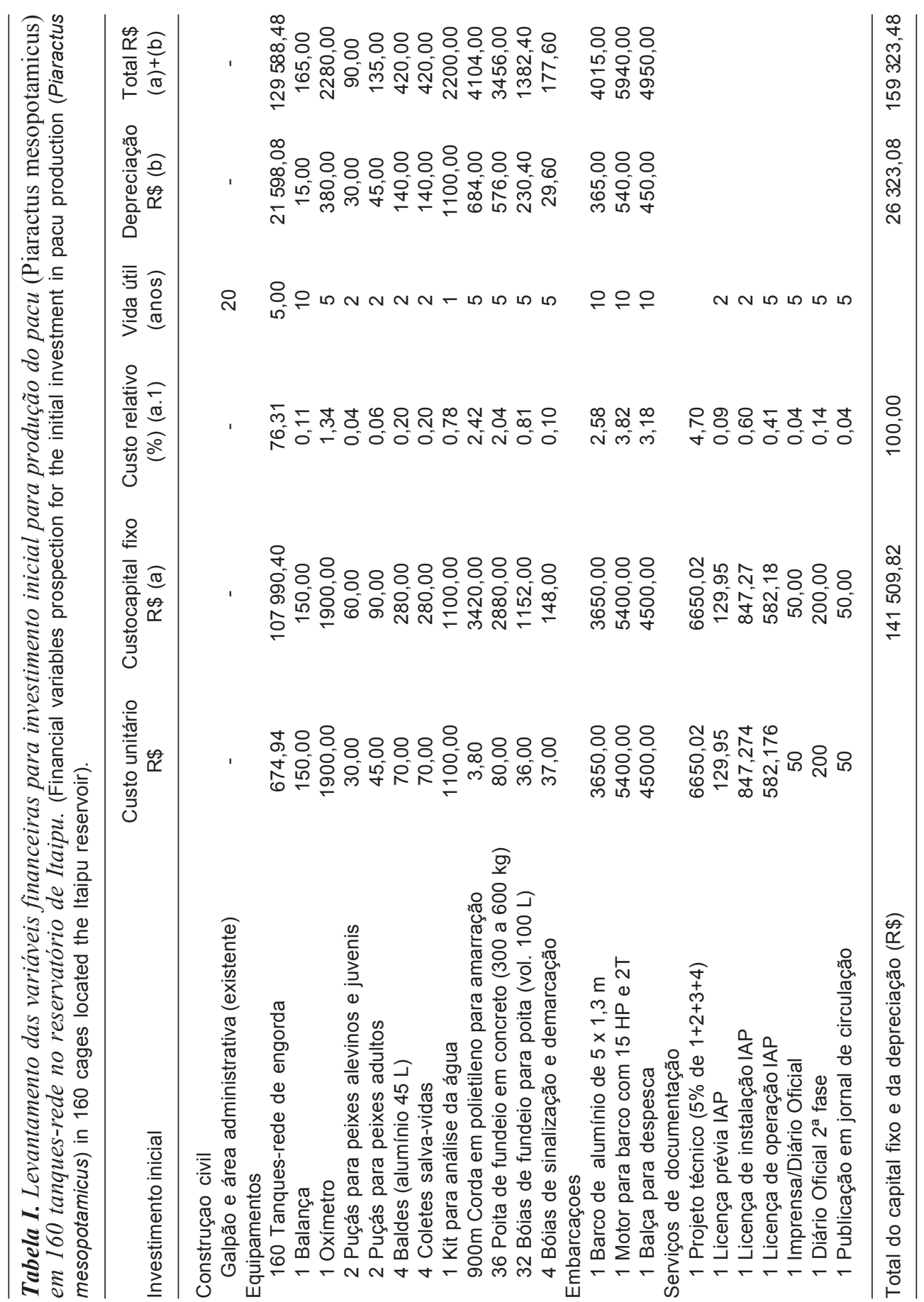

Archivos de zootecnia vol. 61, núm. 234, p. 249. 
panema, estado de São Paulo, Furlaneto et al. (2006) produzindo tilápia do Nilo (Oreochromis niloticus) encontraram uma rentabilidade mínima de $173 \mathrm{t} / \mathrm{ciclo} / \mathrm{ha}$, atingindo $10 \%$ de lucratividade em 6 meses. Valores acima dos obtidos pelos sistemas tradicionais de cultivo de peixes tropicais em viveiros escavados, que quando bem manejados, representam rentabilidade de 12,7\% em 12 meses (Crivelenti et al., 2006). Tal fato pode ser explicado devido as maiores densidades empregadas e o melhor aproveitamento das dietas pelos peixes confinados, as condições ambientais do local e a biomassa produtiva utilizada por ciclo de produção que tornam-se delimitadores do tempo de retorno do investimento e da capacidade de suporte do ambiente, fatores que correlacionam-se a sustentabilidade do sistema produtivo (Kubitza, 1999). Estes fatores podem ser observados na tabela II, quando simulado os cenários mais pessimistas $(\mathrm{G}, \mathrm{H}, \mathrm{I}$ e $\mathrm{J})$ onde o sistema produtivo obteve um déficit na sua lucratividade e no retorno sobre o investimento(ROI). Esta projeção dos índices econômicos também foram relatados por Marengoni e Bueno (2007) avaliando a rentabilidade da tilápia do Nilo em tanques de $4 \mathrm{~m}^{3}$ durante 135 dias sob as densidades 250, 300, 350 e 400 peixes $/ \mathrm{m}^{3}$ obtiveram o melhor lucro de $41,50 \mathrm{R} \$ / \mathrm{m}^{3}$ para a densidade intermediária de 300 peixes $/ \mathrm{m}^{3}$.

De acordo com Ostrensky et al. (2008) a rentabilidade e o retorno econômico na produção de peixes diferenciam-se de acordo com o sistema de cultivo o qual o empreendedor irá utilizar, podendo este ser intensivo, extensivo ou semi-intensivo.

Um dos principais fatores limitantes no cultivo de peixes está relacionado ao custo com a ração e segundo Andrade et al. (2005), a ração corresponde a 52,19\% do custo de produção. Para Crivelenti et al. (2006), este item contribui com $41,07 \%$ no custo total. Entretanto, a utilização de novas estratégias de manejo alimentar no cultivo de peixes contribuirá com a queda nos custos com a alimentação, ocasionando a melhor lucra- tividade dentro do empreendimento aquícola (Kubitza e Ono, 2004). Na maioria dos casos, o produtor adere aos modelos pré-estabelecidos pelas empresas de rações e às orientações dos técnicos do ramo, os quais geralmente não fazem o planejamento zootécnico específico para cada realidade, ocasionando a menor rentabilidade do sistema de cultivo, desperdício de estrutura e maior poluição do ambiente. As projeções de cenários mais pessimistas estão representadas nos itens de E a J na tabela II, diminuindo assim as possibilidades deste produtor de tornar-se competitivo dentro dos novos moldes da aqüicultura mundial (Eler e Millani, 2007). Furuya (2007) e Boscolo et al. (2008) vêm demonstrando que algumas estratégias no manejo nutricional dos peixes como a formulação de rações através do conceito de proteína ideal, da consideração do valor nutritivo dos alimentos, determinação da exigências para cada espécie, redução da proteína da dieta, melhora do processamento, uso de prónutrientes e fornecimento mínimo de fósforo disponível trazem maior eficiência dos peixes. Relacionando-se a conversão alimentar (CA), ganho de peso diário, declinando o tempo de cultivo que irá perfazer maior retorno econômico sobre o tempo do investimento, além de menor emissão de efluentes.

De acordo com Signor et al. (2010), a CA do pacu criado em tanques-rede apresentaram valores médios de 3,34; 3,51 e 3,34. Carneiro et al. (1992) encontrou conversão alimentar de 1,54 a 2,01 para diferentes faixas de peso de pacu, valores próximos ao de Scorvo Filho et al. (1998) que obtiveram em média uma CA de 1,80 para tilápia.

Na tabela II, verifica-se que nos cenários A e B com a CA de 2,92 e custo da ração de $\mathrm{R} \$ 0,70$, os valores de COE, COT e ponto de equilíbrio financeiro foram semelhantes. $\mathrm{O}$ ponto nivelador do retorno econômico entre estes dois cenários (A e B) foi o preço de venda do peixe, que ao aumentar em 0,15 centavos proporcionou um acréscimo de 9 
RETORNO ECONÔMICO DA PRODUÇÃO DE PACU EM TANQUES-REDE

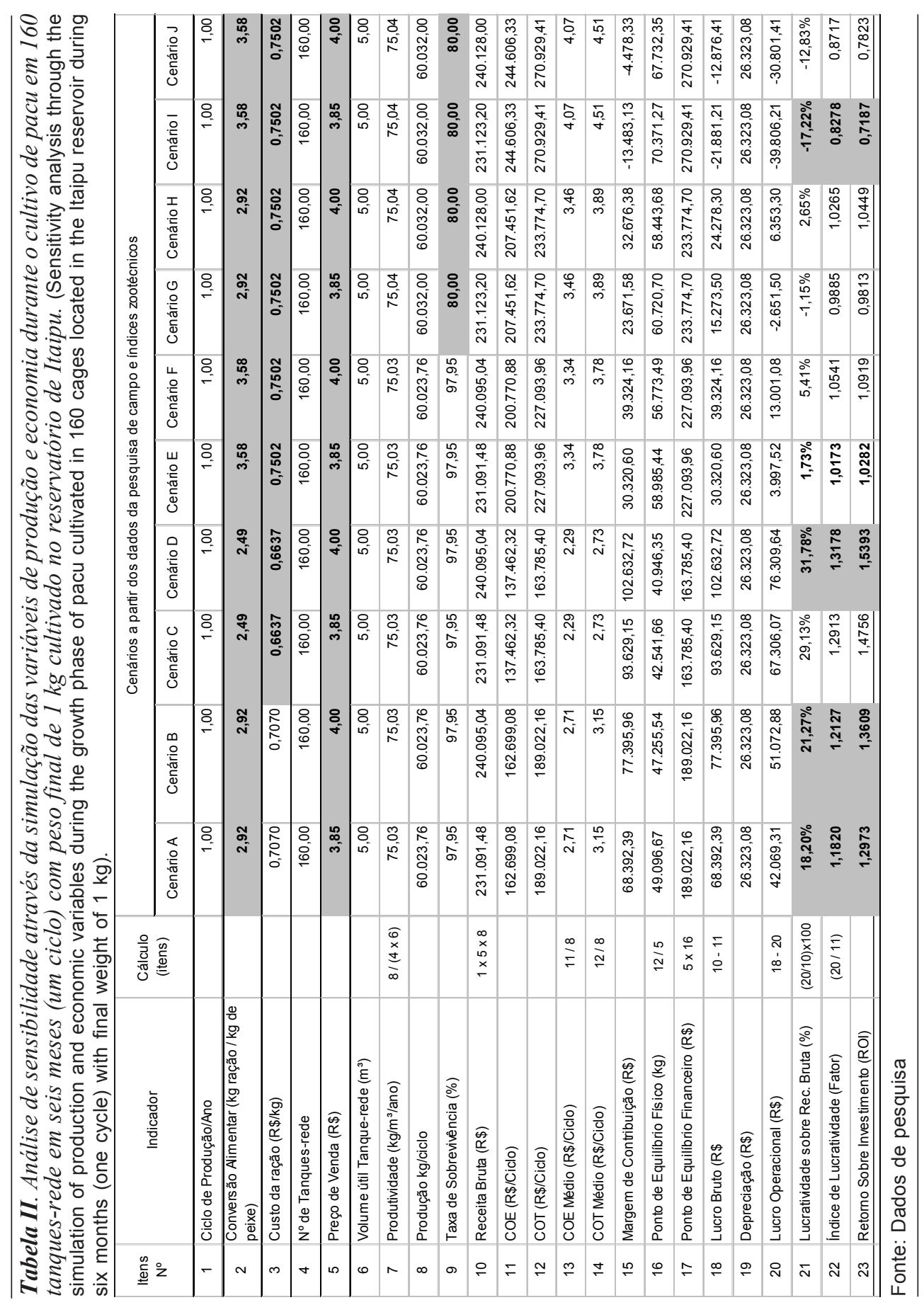

Archivos de zootecnia vol. 61, núm. 234, p. 251. 


\section{SILVA, RABENSCHLAG, FEIDEN, BOSCOLO, SIGNORE BUENO}

mil reais na margem de contribuição alterando o ponto de equilíbrio positivamente em $11,63 \%$. Ou seja, o fator preço de venda do peixe ocasionou um incremento na lucratividade sobre a receita bruta de $2,53 \%$ maior no cenário $\mathrm{B}$ em relação ao $\mathrm{A}$. Esta proporção também pode ser vista nos cenários $\mathrm{C}$ e $\mathrm{D}$, os quais foram simulados com o menor custo com ração $0,6637 \mathrm{R} \$ / \mathrm{kg}$ e CA de 2,49, com isto verificou-se que o índice de lucratividade elevou-se a medida que o preço de venda do peixe aumentou e consequentemente o retorno sobre o investimento foi de 1,3609 $\mathrm{R} \$ / \mathrm{kg}$.

Diante das projeções realizadas, os cenários E, F, G, H, I e J são os mais pessimistas dentro da análise determinística. Estipulou-se valores menos eficientes para conversão alimentar, custo com ração e taxa de sobrevivência. Com isso, a lucratividade sobre a receita bruta não ultrapassou 5,41\% representada no cenário $\mathrm{F}$, obtendo o pior índice de $-17,22 \%$ no cenário I. Isto deve-se a elevada conversão alimentar considerada, custo com a ração, além do preço de venda do peixe mais baixo e da taxa de sobrevivência que reduziu-se para $80 \%$. Pois quando estocado juvenis com peso médio de $293,38 \pm 5,67 \mathrm{~g}$, como o utilizado neste estudo, apenas em casos muito pessimista o risco de mortalidade irá ultrapassa $20 \%$.

A análise de retorno sobre o investi-

\section{BIBLIOGRAFIA}

Andrade, R.L.B., Wagner, R.L., Mahl, I. e Martins, R.S. 2005. Custos de produção de tilápias (Oreochromis niloticus) em um modelo de propriedade da região oeste do Estado do Paraná, Brasil. Cienc Rural, 35: 198-203.

Ayroza, L.M.S. 2009. Criação de Tilápia-do-Nilo, Oreochromis niloticus, em tanques-rede, na Usina Hidrelétrica de Chavantes, Rio Paranapanema. Tese (doutorado). Universidade Estadual Paulista. Centro de Aquicultura. Jaboticabal. $92 \mathrm{pp}$.

Boscolo, W.R., Hayashi, C., Feiden, C., Meurer, F. e Signor, A.A. 2008. Composição química e mento (ROI) demonstrou que as melhores projeções encontram-se para os índices de 1,$2973 ; 1,3609 ; 1,4756 ; 1,5993 \mathrm{R} \$ / \mathrm{kg}$ nos cenários $\mathrm{A}$ a $\mathrm{D}$ resaltando que para o empreendedor que pretende investir no agronegócio do peixe cada um real investido é possível obter um ganho de 0,30 a 0,54 centavos.

\section{CONCLUSÃO}

O retorno econômico da produção de pacu em tanques-rede no reservatório de Itaipu demonstrou que os empreendedores que pretendem investir no agronegócio piscícola devem produzir no mínimo 40 946,35 $\mathrm{kg}$ de pacu em 160 tanques-rede com uma conversão alimentar de 1:2,49 e preço de venda do peixe a $4,00 \mathrm{R} \$ / \mathrm{kg}$ para atingir $31,78 \%$ de lucratividade sobre a receita bruta obtendo retorno econômico de 1,5393 R \$/ $\mathrm{kg}$ sobre o investimento. Ressaltando ainda que existe a possibilidade de um retorno de $18 \%$ a $31 \%$ sobre a receita bruta.

\section{AGRADECIMENTOS}

Ao convênio AS/CT/0100/05 firmado entre o Ministério da Pesca e Aquicultura, Itaipu Binacional, Fundação Universitária de Toledo e Universidade Estadual do Oeste do Paraná, pela estrutura e insumos fornecidos para a realização do experimento.

digestibilidade aparente da energia e nutrientes da farinha de resíduos da indústria de filetagem de tilápias, para a tilápia do Nilo (Oreochromis niloticus). Cienc Rural, 38: 2579-2586.

Carneiro, D.J., Wagner, P.M., Dias, T.C.R. e Carvalho, D.D.G. 1992. Efeito da densidade de estocagem e do nível de proteína bruta no desempenho de produção do pacu (Piaractus mesopotamicus). Resultados preliminares. Simpósio Brasileiro de Aqüicultura, 7. Anais... SIMBRAQ. Peruíbe, SP. pp. 14.

Castagnolli, N. e Zuim, S.M.F. 1985. Consolidação do conhecimento adquirido sobre Colossoma 


\section{RETORNO ECONÔMICO DA PRODUÇÃO DE PACU EM TANQUES-REDE}

mitrei (Berg, 1985). FCAV/Unesp. Jaboticabal.

Conte, L. 2002. Produtividade e economicidade da tilapicultura em gaiolas na região sudoeste do estado de São Paulo: Estudos de casos. Dissertação de Mestrado em Agronomia (Ciência Animal e Pastagens). Universidade de São Paulo. ESALQ/USP. Piracicaba. 59 pp.

Crivelenti, L.Z., Borin, S., Pirtouscheg, A., Neves, J.E.G. e Abdão, E.M. 2006. Desempenho econômico da criação de tilápias do Nilo (Oreochromis niloticus) em sistema de produção intensiva. Rev Vet Notícias, 12: 117122.

Dillon, P.J. and Rigler, F.H. 1974. Thephosphoruschlorophyllrelationshipin lakes. Limnol Oceanogr, 19: 767-773.

Eler, M.N. e Millani, T.J. 2007. Métodos de estudos de sustentabilidade aplicados á aqüicultura Rev Bras Zootecn, 36: 33-44.

Faria, R.H.S., Souza, M.L.R., Wagner, P.M., Povh, J.A. e Ribeiro, R.P. 2003. Rendimento do processamento da tilapia do Nilo (Oreochroms niloticus Linnaeus, 1757) e do pacu (Piaractus mesopotamicus Holmberg, 1887). Acta Sci Anim Sci, 25: 21-24.

FAO. 2010. Food and Agriculture Organization. The State of World Fisheries and Aquaculture FAO. Rome. 197 pp.

Filipetto, J.E.S. 2004. Normas para instalação de pisciculturas. Em: B. Baldisserotto, J. Radünz Neto (Orgs.). Criação de jundiá. $1^{\mathrm{a}}$ ed. Editora da UFSM. Santa Maria. pp. 13-34.

Furuya, W.M. 2007. Redução do impacto ambiental por meio de ração. Seminário de Aves e Suínos 7. Anais... Avesui. Belo Horizonte. pp.121-139.

Furlaneto, F.P.B., Ayroza, D.M.M.R. e Ayroza, L.M.S. 2006. Custo e rentabilidade da produção de tilápia (Oreochromis ssp.) em tanque-rede no médio Paranapanema, estado de São Paulo, Safra 2004/2005. Informações Econômicas, SP, 36: 63-69.

Godinho, H.P. 2007. Estratégias reprodutivas de peixes aplicada à aquicultura: bases para 0 desenvolvimento de tecnologias de produção. Rev Bras Rep Anim, 31: 351-360.

IAP-Instituto Ambiental do Paraná. 2010. Licenciamento e autorização. www.iap.pr.gov.br (15/ 01/2010).

Itaipu Binacional. 2007. Plano Diretor do Reservatório de Itaipu. http://www.itaipu. gov.br (12/10/2010).
Jomori, R., Carneiro, D.J. and Portella, M.C. 2003. Growth and survival of pacu Piaractus mesopotamicus (Holmberg, 1887) juveniles reaed in ponds or at different initial larviculture periods indoors. Aquaculture, 221: 277-287.

Kubitza, F. 1999. Tanques-rede, rações e impacto ambiental. Rev Panorama Aquicult, 51: 44-50.

Kubitza, F. e Ono, E.A. 2004. Projeto aqüícolas: planejamento e avaliação econômica. Cultivo de peixes em tanques-rede. $1^{\text {a }}$ ed. F. Kubitza. Jundiaí. 87 pp.

Kubitza, F. e Campos, J.L. 2005. Desafios para a consolidação da tilapicultura no Brasil. Rev Panorama Aquicult, 15: 14-21.

Marengoni, N.G. e Bueno, G.W. 2007. Viabilidade econômica na produção de tilápias utilizando diferentes biomassas em tanques rede no reservatório da UHE de Rosana-SP. Congresso Internacional de Zootecnia, 4., Congresso Brasileiro de Zootecnia, 17. Anais... UEL. Londrina.

Matsunaga, M., Bemelmans, P.F., Toledo, P.E.N., Dulley, R.D., Okawa, H. e Pedroso, I.A. 1976. Metodologia de custo de produção utilizada pelo IEA. Agr São Paulo, 23: 123-139.

Medeiros, F.C. 2002. Tanque-rede: mais tecnologia e lucro na piscicultura. Centro América. Cuiabá. $110 \mathrm{pp}$.

MPA-Ministério da Pesca e Aquicultura. 2010. Estatística da pesca e aquicultura no Brasil 2008/2009. http://www.mpa.gov.br/\#infoestatistica/estatistica-da-pesca-e-aquicultura (25-11-2010).

Ono, E.A. e Kubitza, F. 2003. Cultivo de peixes em tanques-rede. Jundiaí. $112 \mathrm{pp}$.

Ostrensky, A., Borghetti, J.R. e Sato, D. 2008. Aqüicultura no Brasil: o desafio é crescer. Brasília. 276 pp.

Petrere, J.R.M. 1989. River fisheries in Brazil: A review. Regul River, 4: 1-16.

Richter, G.O. 2004. Pesca e aqüicultura. Secretaria de Estado e do Abastecimento (SEAB/ DERAL). Departamento de Economia RuralDivisão de Conjuntura Agropecuária do Estado do Paraná (DCA). Curitiba. 35 pp.

Signor, A.A., Boscolo, W.R., Bittencourt, F., Coldebella, A. e Reidel, A. 2010. Proteína e energia na alimentação de pacus criados em tanques-rede. Rev Bras Zootecn, 39: 23362341.

Archivos de zootecnia vol. 61, núm. 234, p. 253. 


\section{SILVA, RABENSCHLAG, FEIDEN, BOSCOLO, SIGNORE BUENO}

Scorvo-Filho, J.D., Martin, N.B. e Ayroza, L.M.S. 1998. Piscicultura em São Paulo: custo e retornos de diferentes sistemas de produção na safra de 1996/1997. Informações Econômicas, 28: 41-61.
Scorvo-Filho, J.D., Pinto, C.S.R.M., Verani, J.R. e Silva, A.L. 2006. Custo operacional de produção da criação de tilápias vermelha da flórida e tailandesa em tanques-rede de pequeno volume. Informações Econômicas, 36: 71-79. 